\title{
HARMONIC MAPS TO TEICHMÜLLER SPACE
}

\author{
Georgios Daskalopoulos, Ludmil Katzarkov, and Richard \\ WENTWORTH
}

\begin{abstract}
We give sufficient conditions for the existence of equivariant harmonic maps from the universal cover of a Riemann surface $B$ to the Teichmüller space of a genus $g \geq 2$ surface $\Sigma$. The condition is in terms of the representation of the fundamental group of $B$ to the mapping class group of $\Sigma$. The metric on Teichmüller space is chosen to be the Kähler hyperbolic metric. Examples of such representations arise from symplectic Lefschetz fibrations.
\end{abstract}

\section{Introduction}

Let $B$ be a closed, compact Riemann surface, and let $\Gamma_{g}$ denote the mapping class group for a closed, oriented, genus $g \geq 2$ surface $\Sigma$. A representation $\rho: \pi_{1}(B) \rightarrow \Gamma_{g}$ gives a homotopy class of $\rho$-equivariant continuous maps $u$ : $\widetilde{B} \rightarrow T_{g}$. Here, $T_{g}$ is the Teichmüller space of equivalence classes of conformal structures on $\Sigma$, and $\widetilde{B}$ denotes the universal cover of $B$.

This paper concerns the following question: for which representations $\rho$ does there exist a $\rho$-equivariant harmonic map $u: \widetilde{B} \rightarrow T_{g}$ ? To give this meaning, we must choose a $\Gamma_{g}$-invariant metric on $T_{g}$. The two most widely studied metrics, and the ones most useful in terms of applications, are the Weil-Petersson and Teichmüller metrics (see $\S 3$ for a brief description). If the Weil-Petersson metric on $T_{g}$ were a complete metric of nonpositive curvature, we could select it for the target metric, and an existence theorem would follow from what are by now standard methods (cf. [D1, C1, La, JY1]). While the Weil-Petersson metric does have strictly negative sectional curvature $[\mathrm{T}, \mathrm{R}, \mathrm{W} 1]$ and is geodesically convex [W2], it is not complete [W3, Chu], and this complicates the analysis (see [JY2, JY3] for a discussion of this). Alternatively, one may attempt to adapt the recent techniques on general metric space targets [GS, KS, J1] to study equivariant maps to $T_{g}$ with respect to the Teichmüller metric. This is only a Finsler metric, but it does give $T_{g}$ the structure of a complete length space. However, it is known that the Teichmüller metric is not nonpositively curved $[\mathrm{M}]$, and therefore the methods of $[\mathrm{KS}]$, and in particular the existence theorems, do not directly apply.

Received June 28, 1999. Revised October 15, 1999.

1991 Mathematics Subject Classification. Primary: 58E20 ; Secondary: 32G13, 30F60, $57 \mathrm{M} 60$.

G.D. supported in part by NSF grant DMS-9803606.

L.K. supported in part by NSF grant DMS-9700605 and a Sloan Research Fellowship.

R.W. supported in part by NSF grant DMS-9971860. 
Recently, McMullen proved that $T_{g}$ carries a complete Kähler metric $g_{1 / \ell}$ that interpolates between the Weil-Petersson and Teichmüller metrics in such a way as to maintain a bounded geometry. More precisely, we have the following

Theorem 1.1 (McMullen $[\mathrm{McM}])$. There is a complete $\Gamma_{g}$-invariant Kähler metric $g_{1 / \ell}$ on $T_{g}$ satisfying the following:

1. $g_{1 / \ell}$ is uniformly quasi-isometric (comparable) to the Teichmüller metric;

2. $\left(\mathcal{M}_{g}, g_{1 / \ell}\right)$ has finite volume, where $\mathcal{M}_{g}=T_{g} / \Gamma_{g}$ is the Riemann moduli space;

3. The sectional curvature of $g_{1 / \ell}$ is bounded from above and below;

4. The injectivity radius of $g_{1 / \ell}$ is bounded from below.

Indeed, this metric satisfies the stronger condition of being Kähler hyperbolic in the sense of Gromov, though we will not need this fact. The construction in $[\mathrm{McM}]$ actually produces a family $g_{1 / \ell}(\varepsilon)$ of such metrics depending upon a sufficiently small parameter $\varepsilon>0$, and $g_{1 / \ell}(\varepsilon)$ converges uniformly on compact sets to the Weil-Petersson metric as $\varepsilon \rightarrow 0$. It is therefore natural to study the harmonic map problem with respect to $g_{1 / \ell}(\varepsilon)$ and the dependence of its solutions on $\varepsilon$. Here we shall only address the first issue. The results in this paper will be valid for any choice of $\varepsilon$, and we henceforth suppress this dependence from the notation.

It will be useful to generalize the problem to the case where the domain is a compact surface with several points removed: $B^{\prime}=B \backslash\left\{p_{1}, \ldots, p_{k}\right\}$. In this case, the given data is a representation $\rho: \pi_{1}\left(B^{\prime}\right) \rightarrow \Gamma_{g}$, and we must assume the existence of a smooth initial finite energy $\rho$-equivariant map $f: \widetilde{B}^{\prime} \rightarrow T_{g}$. As is customary in the equivariant problem, since the energy density $e(f)$ of $f$ is $\pi_{1}\left(B^{\prime}\right)$-invariant, finite energy means that the integral $E(f)$ of $e(f)$ over $B^{\prime}$ is finite. A condition sufficient for the existence of a finite energy map, and analogous to the one for linear representations, is the requirement that the local monodromy about each point $p_{i}$ be pseudoperiodic (see Definition 3.1 and Theorem 3.2 below).

Let $\mathcal{P} \mathcal{M F}$ denote the space of projective classes of measured foliations. Recall that the action of $\Gamma_{g}$ extends continuously to Thurston's compactification $\bar{T}_{g}=$ $T_{g} \cup \mathcal{P} \mathcal{M F}$. A subgroup is sufficiently large if it fixes no finite set of points on the boundary $\mathcal{P} \mathcal{M F}$ (for the precise condition, see Definition 2.2 and Theorem 2.3 below). The main result of this note is the following

Theorem 1.2. Let $T_{g}$ be equipped with the metric $g_{1 / \ell}$. If $B$ is a compact surface and the image of $\rho: \pi_{1}(B) \rightarrow \Gamma_{g}$ is either finite or sufficiently large, then there exists a $\rho$-equivariant harmonic map $u: \widetilde{B} \rightarrow T_{g}$. Similarly, if $\rho: \pi_{1}\left(B^{\prime}\right) \rightarrow \Gamma_{g}$ is either finite or sufficiently large and there exists a finite energy $\rho$-equivariant map $f: \widetilde{B}^{\prime} \rightarrow T_{g}$, then there exists a finite energy $\rho$ equivariant harmonic map $u: \widetilde{B}^{\prime} \rightarrow T_{g}$.

In many ways, the metric $g_{1 / \ell}$ combines the advantages of both the WeilPetersson and Teichmüller metrics, and the Thurston boundary seems to play the same role for Teichmüller space as does the ideal boundary for complete 
negatively curved manifolds. Given this, the construction of the harmonic map follows the basic ideas of Morrey, Lemaire, Schoen-Yau, and Sacks-Uhlenbeck [Le, SY, SU]. More generally, one might expect that energy minimizers with respect to $g_{1 / \ell}$ behave similarly to harmonic maps to negatively curved target spaces for higher dimensional domains. In particular, they should enjoy nice regularity and rigidity properties.

By the fundamental work of Donaldson [D2], many examples of subgroups of $\Gamma_{g}$ arise from symplectic 4-manifolds in the form of symplectic Lefschetz fibrations (SLF's for short). These are smooth maps $X \rightarrow B$ that are genus $g$ fibrations off a nonempty set of singular points $\left\{p_{1}, \ldots, p_{k}\right\} \in B$ (see $\S 6$ ). If $B^{\prime}=B \backslash\left\{p_{1}, \ldots, p_{k}\right\}$, then there is a monodromy representation $\rho: \pi_{1}\left(B^{\prime}\right) \rightarrow \Gamma_{g}$ which locally about the points $p_{i}$ is a Dehn twist about a simple closed curve with a preferred sign.

Because of the condition on the local monodromy, one can easily construct finite energy equivariant maps $\widetilde{B}^{\prime} \rightarrow T_{g}$, and one may attempt to find a homotopic harmonic map. We shall show that for SLF's over $S^{2}$, the monodromy $\rho: \pi_{1}\left(B^{\prime}\right) \rightarrow \Gamma_{g}$ is always sufficiently large (see Corollary 6.3). As a consequence, we will prove

Theorem 1.3. Let $X \rightarrow B$ be any symplectic Lefschetz fibration of genus $\geq 2$ over $B=S^{2}$ with monodromy $\rho$. Then there exists a finite energy $\rho$-equivariant harmonic map $u: \widetilde{B}^{\prime} \rightarrow T_{g}$, where $T_{g}$ again has the metric $g_{1 / \ell}$.

This may be regarded as an analogue of a well-known result in the holomorphic category. Recall that a genus $g$ holomorphic Lefschetz fibration over $B$ defines a holomorphic map from $\widetilde{B}^{\prime}$ to $T_{g}$, which is therefore harmonic for any Kähler metric on $T_{g}$. The theorem above shows that existence of a harmonic map is a notion that generalizes to the symplectic category. This is in contrast to the map $\widetilde{B}^{\prime} \rightarrow \mathcal{H}_{g}$, where $\mathcal{H}_{g}$ is the classifying space of Hodge structures on the first cohomology group of the fiber $\Sigma$. Again, in the case of holomophic fibrations, this is holomorphic and therefore harmonic for any Kähler metric on $\mathcal{H}_{g}$. In general, for the canonical metric on $\mathcal{H}_{g}$ it is known that an equivariant map $\widetilde{B}^{\prime} \rightarrow \mathcal{H}_{g}$ can be homotoped to a harmonic map if and only if the image of the monodromy representation on the first cohomology group of the fiber is reductive. On the other hand, there exist SLF's with nonreductive monodromy representations [KPS], so the symplectic analogue of the holomorphic result will not hold in this case.

\section{Background}

In this section, we collect some facts concerning measured foliations and subgroups of the mapping class group.

Let $\mathcal{S}$ denote the set of isotopy classes of simple closed essential curves on $\Sigma$. For $\sigma \in T_{g}$ and $\gamma \in \mathcal{S}$, we let $\ell_{\gamma} \sigma$ denote the length of the geodesic in $\gamma$ with respect to the hyperbolic metric on $\Sigma$ with conformal structure in $\sigma$ (we will henceforth confuse hyperbolic metrics, their conformal structures, and their equivalence classes, and denote all by $\sigma$ ). If $\alpha, \beta \in \mathcal{S}$, the geometric 
intersection number, $i(\alpha, \beta)$, is the minimal cardinality of the set $\alpha \cap \beta$ as $\alpha$ and $\beta$ range through their isotopy classes.

Let $\mathcal{M F}$ denote the space of measured foliations on $\Sigma$, modulo isotopy and Whitehead equivalence (cf. [FLP, Exposé 5]). We do not consider the "zero measure" to be an element of $\mathcal{M F}$. We say that $F, F^{\prime} \in \mathcal{M F}$ are projectively equivalent if $F^{\prime}$ is obtained from $F$ by scaling the measure by a real number $r>0$. In this case, we write $F^{\prime}=r F$. Let $\mathcal{P} \mathcal{M F}$ denote the projective equivalence classes of elements of $\mathcal{M F}$. For $F \in \mathcal{M F}$ and $\gamma \in \mathcal{S}$, we have an intersection number $i(F, \gamma)$ which is the total measure of a representative of $\gamma$ that is quasi-transverse to $F$. This endows $\mathcal{M F}$ and $\mathcal{P} \mathcal{M F}$ with a topology.

Unbounded sequences $\sigma_{j} \in T_{g}$ have subsequences converging in $\mathcal{P} \mathcal{M} \mathcal{F}$ in the following sense: there are positive numbers $r_{j} \rightarrow 0$ and $F \in \mathcal{M F}$ such that for each $\gamma \in \mathcal{S}, r_{j} \ell_{\gamma} \sigma_{j} \rightarrow i(F, \gamma)$. Any other sequence $r_{j}^{\prime}$ for which $r_{j}^{\prime} \ell_{\gamma} \sigma_{j}$ converges on all $\gamma$ (and not identically to zero) gives rise to a measured foliation that is projectively equivalent to $F$. We shall denote this convergence by $r_{j} \sigma_{j} \rightarrow F$.

For any $\gamma \in \mathcal{S}$ and $r>0$, there is an associated $r \gamma \in \mathcal{M F}$ such that $i(r \gamma, \alpha)=r i(\gamma, \alpha)$ for all $\alpha \in \mathcal{S}$. An important fact is that the intersection number for curves and between curves and measured foliations extends continuously to a nonnegative function $i(\cdot, \cdot)$ on $\mathcal{M F} \times \mathcal{M F}$ such that $i\left(r F, r^{\prime} F^{\prime}\right)=r r^{\prime} i\left(F, F^{\prime}\right)$. We say that $F$ and $F^{\prime}$ are transverse if $i\left(F, F^{\prime}\right) \neq 0$.

We will need the following

Lemma 2.1. Let $\sigma_{j} \in T_{g}$ be an unbounded sequence, and let $\gamma_{j} \in \mathcal{S}$. Suppose there is a constant $L>0$ such that $\ell_{\gamma_{j}} \sigma_{j} \leq L$ for all $j$. Also, assume there are positive numbers $r_{j} \rightarrow 0$ and $s_{j} \leq 1$ such that $r_{j} \sigma_{j} \rightarrow F$ and $s_{j} \gamma_{j} \rightarrow F^{\prime}$ in $\mathcal{M F}$. Then $i\left(F, F^{\prime}\right)=0$.

Proof. Fix a conformal structure $\sigma_{0}$ on $\Sigma$. For any other conformal structure $\sigma_{j}$ on $\Sigma$ there is a conformal hyperbolic metric (also denoted $\sigma_{j}$ ). Let $\varphi_{j}$ denote the Hopf differential for the unique harmonic map $u:\left(\Sigma, \sigma_{0}\right) \rightarrow\left(\Sigma, \sigma_{j}\right)$ in the component of the identity. More precisely, $\varphi_{j}$ is the $(2,0)$-part of the pull-back metric $u^{*} \sigma_{j}$. A calculation shows that $\varphi_{j}$ is a holomorphic quadratic differential on $\left(\Sigma, \sigma_{0}\right)$. Let $V_{\varphi_{j}}$ denote the vertical measured foliation of $\varphi_{j}$. Then by the main result of [Wo], $r_{j} \sigma_{j} \rightarrow F$ implies that $r_{j} V_{\varphi_{j}}$ converges in $\mathcal{M F}$ to a measured foliation that is projectively equivalent to $F$. Up to rescaling, we may assume that $r_{j} V_{\varphi_{j}} \rightarrow F$. Since the intersection number is continuous on $\mathcal{M F} \times \mathcal{M F}$, we have $i\left(F, F^{\prime}\right)=\lim i\left(r_{j} V_{\varphi_{j}}, s_{j} \gamma_{j}\right)$. On the other hand, $i\left(V_{\varphi_{j}}, \gamma\right) \leq \ell_{\gamma} \sigma_{j}$ for any $\gamma \in \mathcal{S}$ (cf. [Wo, Lemma 4.6]). In particular,

$$
i\left(r_{j} V_{\varphi_{j}}, s_{j} \gamma_{j}\right)=r_{j} s_{j} i\left(V_{\varphi_{j}}, \gamma_{j}\right) \leq r_{j} s_{j} \ell_{\gamma_{j}} \sigma_{j} \leq r_{j} L \longrightarrow 0 .
$$

This proves the lemma.

Recall Thurston's classification of surface diffeomorphisms (cf. [FLP]). An element $h \in \Gamma_{g}$ is called reducible if $h$ fixes a collection $\left\{\gamma_{1}, \ldots, \gamma_{m}\right\}$ of isotopy classes of disjoint simple closed essential curves in $\Sigma$. It is called pseudoAnosov if there is $r>1$ and transverse measured foliations $F_{+}, F_{-}$such that $h\left(F_{+}\right)=r F_{+}$, and $h\left(F_{-}\right)=r^{-1} F_{-}$. In this case, the fixed point set of $h$ in $\mathcal{P} \mathcal{M F}$ 
is precisely $\left\{F_{+}, F_{-}\right\}$. The classification states that any $h \in \Gamma_{g}$ is either periodic (finite order), reducible, or pseudo-Anosov, and these are mutually exclusive possibilities.

Definition 2.2 ([MP, p. 142]). A subgroup $\Upsilon \leq \Gamma_{g}$ is sufficiently large if it contains two pseudo-Anosov's with disjoint fixed point sets in $\mathcal{P} \mathcal{M} \mathcal{F}$.

Following $[\mathrm{KM}]$, we introduce the following terminology and notation. For any $F \in \mathcal{P} \mathcal{M F}$, we let

$$
\begin{aligned}
\mathcal{W}_{F} & =\{L \in \mathcal{P} \mathcal{M F}: i(F, L)=0\} \\
\mathcal{Z}_{F} & =\{L \in \mathcal{P} \mathcal{M F}: i(L, \gamma)=0 \Longleftrightarrow i(F, \gamma)=0 \text { for all } \gamma \in \mathcal{S}\} .
\end{aligned}
$$

A measured foliation $F$ is called minimal if $i(F, \gamma)>0$ for every $\gamma \in \mathcal{S}$. Let $\mathcal{F}_{\text {min }} \subset \mathcal{P} \mathcal{M} \mathcal{F}$ denote the subset of projective classes of minimal foliations. The sets $\mathcal{Z}_{F}$ give a partition of $\mathcal{P} \mathcal{M} \mathcal{F} \backslash \mathcal{F}_{\text {min }}$, while for $F \in \mathcal{F}_{\text {min }}$, the condition in (2.1) is an equivalence relation. The set $\mathcal{W}_{F}$ consists precisely of those measured foliations that are topologically equivalent to $F$, but whose measures may be different $[\mathrm{Re}]$.

In practice, we will use the following criteria equivalent to the condition for a subgroup to be sufficiently large.

Theorem 2.3 ([KM, Lemma 1.2.1]). For a subgroup $\Upsilon \leq \Gamma_{g}$, the following are equivalent:

1. $\Upsilon$ is sufficiently large;

2. $\Upsilon$ fixes no finite subset of $\mathcal{P} \mathcal{M \mathcal { F }}$;

3. $\Upsilon$ fixes no finite union of the sets $\mathcal{W}_{F}, F \in \mathcal{F}_{\min }$, nor does it fix a finite union of the sets $\mathcal{Z}_{F}, F \in \mathcal{P} \mathcal{M F} \backslash \mathcal{F}_{\text {min }}$.

\section{Finite energy}

The purpose of this section is to give a criterion for the existence of finite energy maps.

Definition 3.1 (cf. $[\mathrm{MM}]$ ). An element $h \in \Gamma_{g}$ is pseudoperiodic if it is either periodic, or it is reducible and periodic on the components of $\Sigma \backslash\left\{\gamma_{1}, \ldots, \gamma_{m}\right\}$.

For linear representations, the condition of locally (quasi-) unipotent monodromy guarantees the existence of finite energy maps (cf. [C2]). The key point is the existence of an invariant horosphere. The following result shows that the analog of this condition for representations into the mapping class group is locally pseudoperiodic.

Theorem 3.2. Let $B^{\prime}=B \backslash\left\{p_{1}, \ldots, p_{k}\right\}$ and $\rho: \pi_{1}\left(B^{\prime}\right) \rightarrow \Gamma_{g}$ be as in $\S 1$. If the local monodromy of $\rho$ about each $p_{i}$ is pseudoperiodic then there exists a finite energy $\rho$-equivariant map $f: \widetilde{B}^{\prime} \rightarrow T_{g}$, where $T_{g}$ has the metric $g_{1 / \ell}$. 
First some facts concerning metrics on $T_{g}$. Recall that the cotangent space $T_{\sigma}^{*} T_{g}$ is identified with the space of holomorphic quadratic differentials on $(\Sigma, \sigma)$. For $\varphi \in T_{\sigma}^{*} T_{g}$, the Teichmüller cometric is given by $\|\varphi\|_{T}=\int_{\Sigma}|\varphi|$. Tangent vectors $T_{\sigma} T_{g}$ are classes of Beltrami differentials $\mu$ (as with conformal structures, we will use the same notation for $\mu$ and its equivalence class). The Weil-Petersson pairing between tangent and cotangent vectors is

$$
(\varphi, \mu)_{W P}=\int_{\Sigma} \varphi \mu
$$

The Teichmüller metric on $T_{\sigma} T_{g}$ satisfies: $\left|(\varphi, \mu)_{W P}\right| \leq\|\mu\|_{T}\|\varphi\|_{T}$. We will denote the distance function on $T_{g}$ associated to $\|\cdot\|_{T}$ by $d_{T}$.

For a tangent vector $\mu \in T_{\sigma} T_{g}$, let $\mu_{h}$ denote the harmonic representative with respect to the hyperbolic metric $\sigma$. Then the Weil-Petersson metric on $T_{g}$ is given by

$$
\|\mu\|_{W P}^{2}=\int_{\Sigma}\left|\mu_{h}\right|^{2} \sigma
$$

We also introduce the $L^{1}$ norms $\|\mu\|_{1}=\int_{\Sigma}|\mu| \sigma$. We have the following

Lemma 3.3. For all $\mu \in T_{\sigma} T_{g},\|\mu\|_{W P}^{2} \leq\|\mu\|_{T}\left\|\mu_{h}\right\|_{1}$.

Proof. We may write $\mu_{h}=\bar{\varphi} \sigma^{-1}$ for a holomorphic quadratic differential $\varphi$. Then

$$
\|\varphi\|_{T}=\int_{\Sigma}|\varphi|=\left\|\mu_{h}\right\|_{1}
$$

On the other hand,

$$
\|\mu\|_{W P}^{2}=\int_{\Sigma}\left|\mu_{h}\right|^{2} \sigma=\int_{\Sigma} \mu\left(\varphi \sigma^{-1}\right) \sigma=\left|(\varphi, \mu)_{W P}\right| \leq\|\mu\|_{T}\|\varphi\|_{T}=\|\mu\|_{T}\left\|\mu_{h}\right\|_{1} .
$$

Proof of Theorem 3.2. Let $p \in B$ be one of the points $p_{i}$. By assumption, the monodromy of $\rho$ about $p$ is pseudoperiodic and so leaves invariant a collection $\left\{\gamma_{1}, \ldots, \gamma_{m}\right\}$ of isotopy classes of simple closed curves. Then according to [MM] we may find a smooth map $f_{p}: \Delta^{*} \rightarrow \mathcal{M}_{g}$, where $\Delta^{*}$ is the punctured disk, such that the monodromy of the lift $\tilde{f}_{p}: \widetilde{\Delta}^{*} \rightarrow T_{g}$ coincides with the local monodromy of $\rho$ at $p$. Note that this map may not be holomorphic (or anti-holomorphic), unless the induced Dehn twists about the $\gamma_{i}$ 's have a coherent sign. Nevertheless, the construction in $[\mathrm{MM}]$ may be adapted so that the polar coordinates $(r, \theta)$ on $\Delta^{*}$ are sent, in terms of Fenchel-Nielson coordinates, to the length and twist parameters of $\gamma_{i}$. More precisely, we may arrange that for $z \in \Delta^{*},|z|$ sufficiently small, the length of $\gamma_{i}$ with respect to the hyperbolic metric in the conformal class $f_{p}(r, \theta)$ is $-1 / \log r$. We may also assume that there are no curves other than $\gamma_{i}$ with length less than $\varepsilon$. Furthermore, the vector field $\left(f_{p}\right)_{*}(\partial / \partial \theta)$ is a linear combination with constant coefficients of the twist vector fields about the $\gamma_{i}$. 
By [McM, Corollary 5.4], there are constants $C_{1}$ and $C_{2}$ (depending on $\varepsilon$ ) such that for any $\mu \in T_{\sigma} T_{g}$ where $\sigma$ is in the image of $f_{p}(z), 0<|z|<\varepsilon$,

$$
\|\mu\|_{T}^{2} \leq C_{1}\|\mu\|_{W P}^{2}+C_{2} \sum_{i=1}^{m}\left|\left(\partial \log \ell_{\gamma_{i}}\right)(\mu)\right|^{2} .
$$

For the vector $\mu=\left(f_{p}\right)_{*}(\partial / \partial \theta)$, the second term on the right hand side of $(3.1)$ vanishes, and the first term is uniformly bounded by [W5, Lemma 3.1]. For the vector $\mu=\left(f_{p}\right)_{*}(\partial / \partial r)$, the second term is bounded by a constant times $1 / r^{2}(\log r)^{2}$. For the first term, we again refer to [W5, Lemma 3.2], which states the the vector fields $\left(\partial / \partial \ell_{\gamma_{i}}\right)_{h}$ have uniform $L^{1}$ bounds. By Lemma 3.3, we can then absorb the Teichmüller norm to the left hand side of (3.1), introducing a term bounded by a constant times $1 / r^{2}(\log r)^{4}$. Hence, the energy density of $f_{p}$ with respect to the Teichmüller metric is bounded by a constant times $1 / r^{2}(\log r)^{2}$, and so $f_{p}$ has finite energy with respect to the Teichmüller metric. Now $g_{1 / \ell}$ is comparable to the Teichmüller metric, so $f_{p}$ has finite energy with respect to $g_{1 / \ell}$ as well. In this way, we construct finite energy maps $f_{p}$ locally for each puncture $p$. We may then extend their lifts equivariantly to obtain a finite energy map as in the statement.

\section{The Dirichlet problem}

For this section only, $B$ will denote a compact surface with nonempty smooth boundary. Given $\rho: \pi_{1}(B) \rightarrow \Gamma_{g}$, we fix a smooth $\rho$-equivariant map $f: \widetilde{B} \rightarrow T_{g}$. Choose a finite index normal subgroup $\widehat{\Gamma} \leq \Gamma_{g}$ such that $\widehat{\mathcal{M}}_{g}=T_{g} / \widehat{\Gamma}$ is a smooth manifold (cf. [L]). If $H$ denotes the kernel of the map $\pi_{1}(B) \rightarrow \Gamma_{g} / \widehat{\Gamma}$ induced by $\rho, \widehat{B}=\widetilde{B} / H$, and $G=\pi_{1}(B) / H$, then $G$ has an induced representation in $\Gamma_{g} / \widehat{\Gamma}$, and $f$ descends to a $G$-equivariant map $\hat{f}: \widehat{B} \rightarrow \widehat{\mathcal{M}}_{g}$. Since $\widehat{B}$ is a finite cover of $B$, it is also a compact surface with boundary. The goal of this section is to prove the following:

Theorem 4.1. There exists a G-equivariant, harmonic map $\hat{u}: \widehat{B} \rightarrow \widehat{\mathcal{M}}_{g}$ with $\left.\hat{u}\right|_{\partial \hat{B}}=\left.\hat{f}\right|_{\partial B}$. Moreover, $\hat{u}$ is homotopic to $\hat{f}$ rel boundary, and $\hat{u}$ is energy minimizing over all homotopic G-equivariant maps with the same boundary conditions.

Since $\pi_{2}\left(\widehat{\mathcal{M}}_{g}\right)=0$, the proof of Theorem 4.1 follows along the lines of [Le, $\mathrm{SY}, \mathrm{SU}$. However, some additional care is required for two reasons. First, we demand that the maps $\hat{u}$ be $G$-equivariant; and second, the injectivity radius of $g_{1 / \ell}$ on $\widehat{\mathcal{M}}_{g}$ is not bounded away from zero. Neither of these points presents a real difficulty, however, since the equivariance is essentially dealt with in the above references, and the injectivity radius of $g_{1 / \ell}$ on the universal cover $T_{g}$ is bounded from below. For the sake of completeness, we provide the necessary modifications to the standard arguments.

The method of proof will closely follow [J2, J3], where the Sacks-Uhlenbeck theorem is proven using harmonic replacements. Here is an outline: take an energy minimizing sequence on $\widehat{B}$ with given boundary data and cover $\widehat{B}$ by 
finitely many disks whose radius is small with respect to the initial data and the geometry of $g_{1 / \ell}$ on $T_{g}$. We then apply the Courant-Lebesgue Lemma (cf. [J3, Lemma 1.3.2]) to replace the maps on each disk by harmonic maps with the same boundary data. In this way, we construct an equicontinuous family from which a subsequence converging to an energy minimizer may be extracted.

First, some notation: we fix a conformal metric on $B$, and all distances on $B$ (and $\widehat{B}$ ) will be taken with respect to this metric. For $x \in \widehat{B}, \varepsilon>0$, we let $\mathcal{D}(x, \varepsilon)$ denote the set of points in $\widehat{B}$ of distance $<\varepsilon$ from $x$. We shall always assume that $\varepsilon$ is sufficiently small so that $\mathcal{D}(x, \epsilon)$ is topologically either a disk or a half-disk (if $x$ is near the boundary). Similarly, for $p \in \widehat{\mathcal{M}}_{g}$, let $\mathcal{B}(p, \varepsilon)$ denote the set of points in $\widehat{\mathcal{M}}_{g}$ of distance $<\varepsilon$ from $p$ with respect to the $g_{1 / \ell}$ metric. Here, we do not make an assumption on the topology of $\mathcal{B}(p, \varepsilon)$.

Let $K=E(\hat{f}), R=\frac{1}{3} \min \left(i\left(T_{g}\right), \pi / 2 \sqrt{\kappa}\right)$, where $i\left(T_{g}\right)$ is the lower bound for the injectivity radius of $T_{g}$ in the $g_{1 / \ell}$ metric, and $\kappa \geq 0$ is the upper bound for the sectional curvature. By Theorem 1.1, $R$ is strictly positive. We also choose a fundamental domain $F$ in $\widehat{B}$ for the action of $G$. Cover $F$ by disks $\mathcal{D}\left(x_{j}, \delta / 2\right)$, $j=1, \ldots, m$, where $0<\delta<1$ is chosen so that

(i) $8 \pi K / \log (1 / \delta) \leq R^{2}$;

(ii) If $d_{1 / \ell}$ denotes the distance with respect to the metric $g_{1 / \ell}$, then $d_{1 / \ell}\left(\hat{f}\left(x_{1}\right), \hat{f}\left(x_{2}\right)\right) \leq R$, for all $x_{1}, x_{2} \in \partial \widehat{B}$ satisfying $d\left(x_{1}, x_{2}\right) \leq \sqrt{\delta}$. This is possible from the compactness of $\partial \widehat{B}$;

(iii) $\gamma \cdot \mathcal{D}\left(x_{j}, \sqrt{\delta}\right) \cap \mathcal{D}\left(x_{j}, \sqrt{\delta}\right)=\emptyset$, for all $j$ and all $\gamma \in G$ different from the identity. This is possible because $G$ is finite acting freely.

Let $\hat{f}_{n}: \widehat{B} \rightarrow \widehat{\mathcal{M}}_{g}$ be a sequence of continuous $G$-equivariant maps such that

(iv) $\left.\hat{f}_{n}\right|_{\partial \hat{B}}=\left.\hat{f}\right|_{\partial \hat{B}}$;

(v) $E\left(\hat{f}_{n}\right)$ converges to the infimum of the energy over all $G$-equivariant maps in the homotopy class of $\hat{f}$ rel boundary.

By the Courant-Lebesgue Lemma (cf. [J3, Lemma 1.3.2]), for every $n$ we can find $r_{n, 1}, \delta<r_{n, 1}<\sqrt{\delta}$ and $p_{n, 1} \in \widehat{\mathcal{M}}_{g}$ such that $\hat{f}_{n}\left(\partial \mathcal{D}\left(x_{1}, r_{n, 1}\right)\right) \subset$ $\mathcal{B}\left(p_{n, 1}, R\right)$. Since the disk is simply connected, the map $\hat{f}_{n}$ lifts to a map $\tilde{f}_{n}: \mathcal{D}\left(\tilde{x}_{1}, r_{n, 1}\right) \rightarrow T_{g}$, and we clearly have $\hat{f}_{n}\left(\mathcal{D}\left(\tilde{x}_{1}, r_{n, 1}\right)\right) \subset \mathcal{B}\left(\tilde{p}_{n, 1}, R\right)$, for $\tilde{p}_{n, 1}$ a lift of $p_{n, 1}$. Let $\tilde{h}_{n}: \mathcal{D}\left(\tilde{x}_{1}, r_{n, 1}\right) \rightarrow T_{g},\left.\tilde{h}_{n}\right|_{\partial \mathcal{D}\left(\tilde{x}_{1}, r_{n, 1}\right)}=\left.\tilde{f}_{n}\right|_{\partial \mathcal{D}\left(\tilde{x}_{1}, r_{n, 1}\right)}$ be harmonic and energy minimizing. It follows by [J3, Lemmas 1.3.3, 1.3.4] that $\tilde{h}_{n}\left(\mathcal{D}\left(\tilde{x}_{1}, r_{n, 1}\right)\right) \subset \mathcal{B}\left(\tilde{p}_{n, 1}, R\right)$, and the $\tilde{h}_{n}$ have uniform modulus of continuity. The maps $\tilde{h}_{n}$ descend to harmonic maps: $\hat{h}_{n}: \mathcal{D}\left(x_{1}, r_{n, 1}\right) \rightarrow \mathcal{B}\left(p_{n, 1}, R\right) \subset \widehat{\mathcal{M}}_{g}$, $\left.\hat{h}_{n}\right|_{\partial \mathcal{D}\left(x_{1}, r_{n, 1}\right)}=\left.\tilde{f}_{n}\right|_{\partial \mathcal{D}\left(x_{1}, r_{n, 1}\right)}$ with the same properties. We now define

$$
\hat{u}_{n}^{1}(x)= \begin{cases}\hat{f}_{n}(x), & \text { if } \quad x \in \widehat{B} \backslash \bigcup_{\gamma \in G} \mathcal{D}\left(\gamma x_{1}, r_{n, 1}\right), \\ \rho(\gamma) \cdot \hat{h}_{n}(x), & \text { if } \quad x \in \gamma \cdot \mathcal{D}\left(x_{1}, r_{n, 1}\right) .\end{cases}
$$

Assumption (iii) implies that $\hat{u}_{n}^{1}$ is well-defined, continuous, and $G$-equivariant. Clearly, we also have $E\left(\hat{u}_{n}^{1}\right) \leq E\left(\hat{f}_{n}\right)$. Furthermore, as in [J2, §4.4], we may 
assume that $r_{n, 1} \rightarrow r_{1}$. By the uniform modulus of continuity, we may assume that $\hat{u}_{n}^{1}$ converge uniformly on $\mathcal{D}\left(x_{1}, \delta-\eta\right)$ and all its translates under $G$, for all $0<\eta<\delta$.

Now we can apply the same argument for $x_{2}$ and obtain maps $\hat{u}_{n}^{2}$ with $E\left(\hat{u}_{n}^{2}\right) \leq$ $E\left(\hat{u}_{n}^{1}\right) \leq E\left(\hat{f}_{n}\right)$. By the uniform modulus of continuity we may assume that the $\hat{u}_{n}^{2}$ converge uniformly on $\mathcal{D}\left(x_{1}, \delta-\eta\right) \cup \mathcal{D}\left(x_{2}, \delta-\eta\right)$ and all its translates, for any $0<\eta<\delta$. We repeat this replacement argument until we obtain a sequence $\hat{u}_{n}^{m}=\hat{v}_{n}$ with $E\left(\hat{v}_{n}\right) \leq E\left(\hat{f}_{n}\right)$ which converges uniformly on all disks $\mathcal{D}\left(x_{i}, \delta / 2\right)$, $i=1, \ldots m$, and their translates. In particular, it converges uniformly on $\widehat{B}$. Let $\hat{v}=\lim \hat{v}_{n}$. Since $T_{g}$ is contractible, each replacement preserves the homotopy type of the map, and $\hat{v}_{n}$ is therefore equivariantly homotopic to $\hat{f}$ with the same boundary values. By lower semicontinuity of the energy we obtain as in [J2, §4.4] that $\hat{v}$ is an energy minimizing harmonic map which is equivariantly homotopic to $\hat{f}$. This completes the proof of Theorem 4.1 .

\section{Proof of Theorem 1.2}

Let $B, B^{\prime}$ be as in $\S 1$, where $B$ is now a compact surface without boundary. We consider the two cases of Theorem 1.2 simultaneously: if $\rho: \pi_{1}(B) \rightarrow \Gamma_{g}$, remove a point $p$ and let $B^{\prime}=B \backslash\{p\}$. Thus, we concentrate on the general problem of a representation $\rho: \pi_{1}\left(B^{\prime}\right) \rightarrow \Gamma_{g}$ and a finite energy $\rho$-equivariant map $f: \widetilde{B}^{\prime} \rightarrow T_{g}$. Let $B_{j}$ be an exhaustion of $B^{\prime}$ by compact surfaces with smooth boundaries. By Theorem 4.1, we may find a sequence of maps $u_{j}: B_{j} \rightarrow \mathcal{M}_{g}$ with the following properties:

1. On a finite cover $\widehat{B}_{j}, B_{j}=\widehat{B}_{j} / G$, the lift $\hat{u}_{j} \rightarrow \widehat{\mathcal{M}}_{g}$ is a $G$-equivariant harmonic map;

2. $\left.\hat{u}_{j}\right|_{\partial \hat{B}_{j}}=\left.\hat{f}\right|_{\partial \hat{B}_{j}}$ and $\hat{u}_{j}$ is homotopic to $\hat{f}$ rel boundary;

3. $\hat{u}_{j}$ is energy minimizing with respect to all $G$-equivariant maps with the same boundary values.

There is another property of the sequence that will be important.

Lemma 5.1. Let $\hat{u}_{j}$ be as above. Then on any compact set $A \subset \widehat{B}^{\prime}$, where $B^{\prime}=\widehat{B}^{\prime} / G$, the $\hat{u}_{j}$ 's have a uniform modulus of continuity.

Proof. Let $K$ be a constant such that $E\left(\hat{u}_{j}\right) \leq K$. For $\varepsilon>0$ choose $0<\delta<1$ such that $8 \pi K / \log (1 / \delta)<\varepsilon^{2}$. By making $\delta$ smaller if necessary, we may assume that $\operatorname{dist}\left(A, \partial \widehat{B}_{j}\right) \geq 2 \sqrt{\delta}$ for $j$ sufficiently large. Given $x \in A$, then by the Courant-Lebesgue Lemma [J3, Lemma 1.3.2] there exists $\delta<r_{j}<\sqrt{\delta}$ for which $\hat{u}_{j}\left(\partial \mathcal{D}\left(x, r_{j}\right)\right) \subset \mathcal{B}\left(p_{j}, \varepsilon\right)$ for some $p_{j} \in \widehat{\mathcal{M}}_{g}$. Since the $\hat{u}_{j}$ are energy minimizers, we have $\hat{u}_{j}(\mathcal{D}(x, \delta)) \subset \hat{u}_{j}\left(\mathcal{D}\left(x, r_{j}\right)\right) \subset \mathcal{B}\left(p_{j}, \varepsilon\right)$ as well (cf. [J3, Lemma 1.3.4]). Now by compactness of $A$ there are finitely many points $x_{1}, \ldots, x_{m}$ such that $A \subset \cup_{i=1}^{m} \mathcal{D}\left(x_{i}, \delta\right)$; hence, for $\eta$ less than the Lebesgue number of this cover, and any $x \in A, \hat{u}_{j}(\mathcal{D}(x, \eta)) \subset \mathcal{B}\left(p_{j}, \varepsilon\right)$, for some $p_{j} \in \widehat{\mathcal{M}}_{g}$. This implies a uniform modulus of continuity. 
We shall say that the sequence $u_{j}$ is bounded if there is a point $x \in \widetilde{B}^{\prime}$ and a subsequence of the maps $\tilde{u}_{j}: \widetilde{B}^{\prime} \rightarrow T_{g}$ such that $\tilde{u}_{j}(x)$ is bounded in $T_{g}$.

Proposition 5.2. If $u_{j}$ is bounded, then there is a subsequence of the maps $\hat{u}_{j}$ converging uniformly on compact sets to a G-equivariant harmonic map $\hat{u}: \widehat{B}^{\prime} \rightarrow \widehat{\mathcal{M}}_{g}$.

Proof. Consider a $G$-invariant precompact domain $\Omega \subset \widehat{B}^{\prime}$ with smooth boundary. We may assume there is a point $x \in \Omega$ such that $\hat{u}_{j}(x)$ is bounded. By Lemma 5.1 and Ascoli's theorem, we may find a subsequence of $\hat{u}_{j}$ converging uniformly on $\bar{\Omega}$ to a map $\hat{u}$. Since $E\left(\hat{u}_{j}\right)$ is uniformly bounded and the $\hat{u}_{j}$ minimize energy with respect to their boundary values, a standard argument shows that $\hat{u}_{i} \rightarrow \hat{u}$ strongly in $L_{1}^{2}$, and $\hat{u}$ is smooth and harmonic on $\Omega$. Since $\hat{u}_{j}$ are $G$-equivariant, so is $\hat{u}$. Finally, by a diagonalization argument, we can arrange for $\hat{u}_{j}$ to converge uniformly on compact sets to some $\hat{u}$ on all of $\widehat{B}^{\prime}$.

Lemma 5.3. If $u_{j}$ is unbounded, then there is a subsequence of the maps $\tilde{u}_{j}$ converging uniformly on compact sets to a $\rho$-equivariant continuous map $\tilde{u}: \widetilde{B}^{\prime} \rightarrow \mathcal{P} \mathcal{M F}$.

Proof. Let $x \in \widetilde{B}^{\prime}$ be such that $\tilde{u}_{j}(x)$ is unbounded. We may choose real numbers $r_{j} \rightarrow 0$ such that some subsequence $r_{j} \tilde{u}_{j}(x)$ converges in $\mathcal{M F}$. Consider $y \in$ $\widetilde{B}^{\prime}$. By Lemma 5.1 there is a uniform bound on $d_{1 / \ell}\left(\tilde{u}_{j}(x), \tilde{u}_{j}(y)\right)$. Since $d_{1 / \ell}$ and $d_{T}$ are comparable, there is a constant $C \geq 0$ independent of $i$ such that $d_{T}\left(\tilde{u}_{j}(x), \tilde{u}_{j}(y)\right) \leq C$. Then by [W4, Lemma 3.1],

$$
e^{-2 C} \ell_{\gamma} \tilde{u}_{j}(y) \leq \ell_{\gamma} \tilde{u}_{j}(x) \leq e^{2 C} \ell_{\gamma} \tilde{u}_{j}(y),
$$

for all $\gamma \in \mathcal{S}$. In particular, a subsequence of $r_{j} \tilde{u}_{j}(y)$ also converges in $\mathcal{M F}$ (note that it suffices to check convergence for finitely many $\gamma$ 's). By a diagonalization argument, we may find a subsequence of $r_{j} \tilde{u}_{j}$ converging in $\mathcal{M F}$ on a countable dense subset of $\widetilde{B}^{\prime}$, and we may do so equivariantly. Again using the equicontinuity, we have that the $r_{j} \tilde{u}_{j}$ converges everywhere to a continuous equivariant $\tilde{u}: \widetilde{B}^{\prime} \rightarrow \mathcal{P} \mathcal{M F}$.

Proposition 5.4. If $u_{j}$ is unbounded, then $\rho$ is not sufficiently large.

Proof. We shall assume that $u_{j}$ is unbounded for $\rho$ sufficiently large and derive a contradiction. Let $\tilde{u}: \widetilde{B}^{\prime} \rightarrow \mathcal{P} \mathcal{M F}$ denote the map obtained from Lemma 5.3. We distinguish two cases:

1. $\tilde{u}\left(\widetilde{B}^{\prime}\right) \cap \mathcal{F}_{\min }=\emptyset$;

2. There is some $x \in \widetilde{B}^{\prime}$ for which $\tilde{u}(x)=F \in \mathcal{F}_{\min }$.

Case 1. Let $\tilde{u}(x)=F \notin \mathcal{F}_{\text {min }}$. We claim that $\tilde{u}(y) \in \mathcal{Z}_{F}$ for all $y \in \widetilde{B}^{\prime}$. In particular, by the equivariance of $\tilde{u}, \mathcal{Z}_{F}$ will be fixed by $\rho$, and this will contradict the assumption of sufficiently large (see Theorem 2.3). To prove the claim, notice that for any $\gamma \in \mathcal{S}$ and some subsequence $u_{j}, i(\tilde{u}(y), \gamma)=\lim r_{j} \ell_{\gamma} \tilde{u}_{j}(y)$. Also, by the uniform bound on $d_{T}\left(\tilde{u}_{j}(x), \tilde{u}_{j}(y)\right),(5.1)$ immediately implies that 
$i(\tilde{u}(y), \gamma)=0$ if and only if $i(\tilde{u}(x), \gamma)=0$; hence, the claim follows from the definition $(2.2)$ of $\mathcal{Z}_{F}$.

Case 2. Assume that $\tilde{u}(x)=F \in \mathcal{F}_{\text {min }}$, and let $\mathcal{W}_{F}$ denote the equivalence class of $F \in \mathcal{F}_{\text {min }}$ as in $(2.1)$. We claim that $\tilde{u}(y) \in \mathcal{W}_{F}$ for all $y \in \widetilde{B}^{\prime}$. By Theorem 2.3 , this will again contradict the assumption of sufficiently large.

Lemma 5.5 (cf. [KM, Lemma 1.4.2]). Let $\sigma_{j}, \sigma_{j}^{\prime} \in T_{g}$ such that $\sigma_{j} \rightarrow F$, $\sigma_{j}^{\prime} \rightarrow F^{\prime}$ in $\mathcal{P} \mathcal{M} \mathcal{F}$, where $F \in \mathcal{F}_{\text {min }}$. Suppose there is a constant $C$ such that for all $j, d_{T}\left(\sigma_{j}, \sigma_{j}^{\prime}\right) \leq C$. Then $F^{\prime} \in \mathcal{W}_{F}$.

Proof. Choose $\gamma_{j} \in \mathcal{S}$ such that $\ell_{\gamma_{j}} \sigma_{j} \leq L$ for some constant $L$ and all $j$. We assume that we have chosen positive numbers $r_{j} \rightarrow 0$ such that $r_{j} \sigma_{j} \rightarrow F$ in $\mathcal{M F}$. We may also find positive numbers $s_{j} \leq 1$ and a subsequence such that $s_{j} \gamma_{j} \rightarrow F^{\prime \prime}$ in $\mathcal{M F}$. Then by Lemma 2.1 we have $i\left(F, F^{\prime \prime}\right)=0$, so $F^{\prime \prime} \in \mathcal{W}_{F}$. On the other hand, the uniform bound on $d_{T}\left(\sigma_{j}, \sigma_{j}^{\prime}\right)$ and (5.1) imply that after passing to a subsequence and possibly rescaling by a nonzero number, $r_{j} \sigma_{j}^{\prime} \rightarrow F^{\prime}$ in $\mathcal{M F}$ as well. In addition, $\ell_{\gamma_{j}} \sigma_{j}^{\prime} \leq e^{2 C} \ell_{\gamma_{j}} \sigma_{j} \leq e^{2 C} L$ for all $j$. Again applying Lemma 2.1, we have $i\left(F^{\prime}, F^{\prime \prime}\right)=0$; so $F^{\prime} \in \mathcal{W}_{F^{\prime \prime}}=\mathcal{W}_{F}$.

Setting $\sigma_{j}=\tilde{u}_{j}(x)$ and $\sigma_{j}^{\prime}=\tilde{u}_{j}(y)$, the claim follows from the lemma, and this completes the proof of Proposition 5.4.

Proof of Theorem 1.2. As above, if $\rho: \pi_{1}(B) \rightarrow \Gamma_{g}$, remove a point $p$ and let $B^{\prime}=B \backslash\{p\}$. Thus, $\rho: \pi_{1}\left(B^{\prime}\right) \rightarrow \Gamma_{g}$, and we assume a finite energy $\rho$-equivariant map $f: \widetilde{B}^{\prime} \rightarrow T_{g}$. If the image $\Upsilon$ of $\rho$ is finite, then by Kerckhoff's solution of the Nielsen Realization Problem $[\mathrm{K}], \Upsilon$ has a fixed point in $T_{g}$. In this case, we may take $\tilde{u}$ to be constant. If $\Upsilon$ is sufficiently large, then we construct a sequence $\hat{u}_{j}$ of harmonic maps for the Dirichlet problems on $\widehat{B}_{j}$ as above. By Proposition 5.4, the $u_{j}$ must be bounded. By Proposition 5.2 we may therefore extract a subsequence converging uniformly on compact sets to a finite energy $\rho$-equivariant harmonic map $\tilde{u}: \widetilde{B}^{\prime} \rightarrow T_{g}$. In case the initial map $f$ extends over $\widetilde{B}$, the uniform modulus of continuity guarantees that the image by $\hat{u}$ of a neighborhood of a puncture $p \in \widehat{B}$ lies in a compact subset of $\widehat{\mathcal{M}}_{g}$. Now by removable singularities [SU, Theorem 3.6] the harmonic map extends, and we obtain an equivariant harmonic map $\tilde{u}: \widetilde{B} \rightarrow T_{g}$.

\section{Lefschetz fibrations}

A genus $g$ Lefschetz fibration is a smooth oriented 4-manifold $X$, a smooth oriented surface $B$, and a smooth map $X \rightarrow B$ which is a fibration by genus $g$ surfaces $\Sigma$ away from finitely many singular fibers at $\left\{p_{1}, \ldots, p_{k}\right\} \subset B$. Furthermore, every critical point has the local description of two complex lines meeting transversely in a double point in such a way that the orientations agree with that of $X$ (see [D2], [GoSt], or [ABKP] for the precise definition). We assume there is at least one critical fiber and at most one critical point per fiber. Thus, the local monodromy of $X$ near a singular fiber is given by a positive Dehn twist 
about a simple closed curve $\gamma \subset \Sigma$, which is called a vanishing cycle. As in $\S 1$, we set $B^{\prime}=B \backslash\left\{p_{1}, \ldots, p_{k}\right\}$.

Gompf showed that under some mild assumptions $X$ may be given a symplectic structure (cf. [GoSt]). In this case, we say $X \rightarrow B$ is a symplectic Lefschetz fibration. Conversely, Donaldson showed that after a finite number of symplectic blow ups, any symplectic 4-manifold admits the structure of an SLF over $S^{2}$ (cf. [D2]). The important thing for us is that for SLF's over $S^{2}$, the product of the positive Dehn twists $D_{\gamma_{1}} \cdots D_{\gamma_{k}}$ describing the local monodromy is the identity in $\Gamma_{g}$.

Recall that a set $\left\{\gamma_{j}\right\}$ of essential simple closed curves is said to fill the surface $\Sigma$ if every essential closed curve $\alpha$ intersects some $\gamma_{j}$ nontrivially (geometrically). We have the following:

Theorem 6.1 (Smith [Sm, Proposition 4.1]). For any symplectic Lefschetz fibration over $S^{2}$, the vanishing cycles fill the fiber.

Proof. Choose a point in the universal cover $\mathbb{H}^{2}$ of the fiber $\Sigma$ and lift each $D_{\gamma_{i}}$ to a $\pi_{1}(\Sigma)$ equivariant diffeomorphism of $\mathbb{H}^{2}$. The extensions $\widehat{D}_{\gamma_{i}}$ of these to the boundary $S^{1} \simeq \partial \mathbb{H}^{2}$ are homeomorphisms which either leave points fixed or move them in a counter-clockwise direction (cf. [K]). Now suppose $\alpha$ is disjoint from the $\gamma_{i}$. We may choose a lift $\tilde{\alpha}$ with two boundary points $p, q \in S^{1}$, and these are necessarily fixed by all $\widehat{D}_{\gamma_{i}}$. The product $\widehat{D}=\widehat{D}_{\gamma_{1}} \cdots \widehat{D}_{\gamma_{k}}$ is therefore monotone, and nonconstant, on each of the components of $S^{1} \backslash\{p, q\}$. On the other hand, $D_{\gamma_{1}} \cdots D_{\gamma_{k}}$ is the identity in $\Gamma_{g}$, and so the restricted lift $\widehat{D}$ must be the restriction of a deck transformation. But the latter are all represented by hyperbolic elements, and so the sign of monotonicity on one of the components of $S^{1} \backslash\{p, q\}$ leads to a contradiction.

Lemma 6.2. Suppose $\Upsilon \leq \Gamma_{g}$ contains a set of Dehn twists $D_{\gamma_{j}}$, where the $\gamma_{j}$ are essential simple closed curves. Assume further that the set $\left\{\gamma_{j}\right\}$ fills the surface $\Sigma$. Then $\Upsilon$ is sufficiently large.

Proof. By Theorem 2.3, it suffices to show that no finite index subgroup $\Upsilon^{\prime}$ of $\Upsilon$ has a fixed point on $\mathcal{P} \mathcal{M F}$. Suppose to the contrary that $F \in \mathcal{P} \mathcal{M F}$ is fixed by $\Upsilon^{\prime}$. We claim that $i\left(F, \gamma_{j}\right)=0$ for all $j$. This would imply that each $\gamma_{j}$ is homotopic to a closed critical leaf of $F$. Since $\left\{\gamma_{j}\right\}$ fills the surface, this would be a contradiction. To prove the claim, note that for any $\gamma_{j}$, there is some power $m_{j}$ such that $D_{\gamma_{j}}^{m_{j}} \in \Upsilon^{\prime}$. If $i\left(F, \gamma_{j}\right) \neq 0$, then by the Picard-Lefschetz formula (cf. [FLP, Exposé 6]),

$$
F=D_{\gamma_{j}}^{m_{j}} F=\lim _{k \rightarrow \infty} D_{\gamma_{j}}^{k m_{j}} F=\gamma_{j}, \text { in } \mathcal{P} \mathcal{M F}
$$

But then, $i\left(\gamma_{j}, \gamma_{j}\right) \neq 0$, contradicting orientability of $\Sigma$.

Combining Lemma 6.2 with Theorem 6.1, we have:

Corollary 6.3. For any symplectic Lefschetz fibration over $S^{2}$, the monodromy representation is sufficiently large. 
Proof of Theorem 1.3. By Corollary 6.3 and Theorem 1.2, it suffices to construct a finite energy $\rho$-equivariant map $f: \widetilde{B}^{\prime} \rightarrow T_{g}$. Since the local monodromies about each of the $p_{i}$ are Dehn twists, and in particular are pseudoperiodic, the result follows from Theorem 3.2.

\section{Acknowledgements}

The authors are grateful to P. Daskalopoulos, T. Pantev, R. Schoen, C. Simpson, I. Smith, A. Stipsicz, and K. Uhlenbeck for discussions. R.W. would like to especially thank Scott Wolpert for pointing out reference [McM]. Finally, we thank the referee for numerous suggestions for improvement.

\section{References}

[ABKP] J. Amorós, F. Bogomolov, L. Katzarkov, and T. Pantev, Symplectic Lefschetz fibrations with arbitrary fundamental group, preprint, GT/9810042.

[Chu] T. Chu, The Weil-Petersson metric in moduli space, Chinese J. Math. 4 (1976), 29-51.

[C1] K. Corlette, Flat G-bundles with canonical metrics, J. Diff. Geom. 28 (1988), 361382 .

[C2] , unpublished.

[D1] S. Donaldson, Twisted harmonic maps and the self-duality equations, Proc. London Math. Soc. (3) 55 (1987), 127-131.

[D2] Lefschetz fibrations in symplectic geometry, Proceedings of the International Congress of Mathematicians, Vol. II (Berlin, 1998), Doc. Math., 1998, Extra Volume II, 309-314.

[FLP] A. Fathi, F. Laudenbach, and V. Poénaru, Travaux de Thurston sur les surfaces, Astérisque, 66-67, Soc. Math. France, Paris, 1979.

[GoSt] R. Gompf and A. Stipsicz, An introduction to 4-manifolds and Kirby calculus, Graduate Studies in Mathematics, 20, American Mathematical Society, Providence, RI, 1999.

[GS] M. Gromov and R. Schoen, Harmonic maps into singular spaces and p-adic superrigidity for lattices in groups of rank one, Inst. Hautes Études Sci. Publ. Math., 76, (1992), 165-246.

[J1] J. Jost, Equilibrium maps between metric spaces, Calc. Var. Partial Differential Equations 2 (1994), 173-204.

[J2] _ Harmonic maps between surfaces, Lecture Notes in Mathematics, 1062, Springer-Verlag, Berlin-New York, 1984.

[J3] Minimal surfaces and Teichmüller theory, in Tsing Hua lectures on geometry and analysis, Hsinchu, Taiwan, 1990-92, International Press, Cambridge, MA, 1995, pp. 149-211.

[JY1] J. Jost and S.-T. Yau, Harmonic maps and group representations, in Differential geometry (H.B. Lawson and K. Tenenblat, eds.), Pitman Monographs Surveys Pure Appl. Math., 52, Longman Sci. Tech., Harlow, 1991, pp. 241-260.

[JY2] $\quad$ On the rigidity of certain discrete groups and algebraic varieties, Math. Ann. 278 (1987), 481-496.

[JY3] - Harmonic mappings and algebraic varieties over function fields, Amer. J. Math. 115 (1993), 1197-1227.

[K] S. Kerckhoff, The Nielsen realization problem, Ann. of Math. 117 (1983), 235-265.

[KM] V. Kaimanovich and H. Masur, The Poisson boundary of the mapping class group, Invent. Math. 125 (1996), 221-264.

[KPS] L. Katzarkov, T. Pantev, and C. Simpson, Nonabelian open orbit theorems, in preparation. 
[KS] N. Korevaar and R. Schoen, Sobolev spaces and harmonic maps for metric space targets, Comm. Anal. Geom. 1 (1993), 561-659.

[La] F. Labourie, Existence d'applications harmoniques tordues à valeurs dans les variétés à courbure négative, Proc. Amer. Math. Soc. 111 (1991), 877-882.

[Le] L. Lemaire, Applications harmoniques de surfaces riemanniennes, J. Differential Geom. 13 (1978), 51-78.

[L] E. Looijenga, Smooth Deligne-Mumford compactifications by means of Prym level structures, J. Algebraic Geom. 3 (1994), 283-293.

[M] H. Masur, On a class of geodesics in Teichmüller space, Ann. of Math. 102 (1975), 205-221.

[MM] Y. Matsumoto and J. Montesinos-Amilibia, Pseudo-periodic homeomorphisms and degeneration of Riemann surfaces, Bull. Amer. Math. Soc. 30 (1994), 70-75.

[McM] C. McMullen, The moduli space of Riemann surfaces is Kähler hyperbolic, preprint, 1999, available at www.math.harvard.edu/ctm

[MP] J. McCarthy and A. Papadopoulos, Dynamics on Thurston's sphere of projective measured foliations, Comment. Math. Helv. 64 (1989), 133-166.

[Re] M. Rees, An alternative approach to the ergodic theory of measured foliations on surfaces, Ergodic Theory Dynamical Systems 1 (1981), 461-488.

[R] H. Royden, unpublished.

[SU] J. Sacks and K. Uhlenbeck, The existence of minimal immersions of 2-spheres, Ann. of Math. 113 (1981), 1-24.

[SY] R. Schoen and S.-T. Yau, Existence of incompressible minimal surfaces and the topology of three-dimensional manifolds with nonnegative scalar curvature, Ann. Math. 110 (1981), 127-142.

[Sm] I. Smith, Symplectic geometry of Lefschetz fibrations, Oxford Thesis, 1999.

[T] A. Tromba, On a natural algebraic affine connection on the space of almost complex structures and the curvature of Teichmüller space with respect to the Weil-Petersson metric, Manuscripta Math. 56 (1986), 475-497.

[Wo] M. Wolf, The Teichmüller theory of harmonic maps, J. Differential Geom. 29 (1989), 449-479.

[W1] S. Wolpert, Chern forms and the Riemann tensor for the moduli space of curves, Invent. Math. 85 (1986), 119-145.

[W2] Geodesic length functions and the Nielsen problem, J. Differential Geom. 25 (1987), 275-296.

[W3] Noncompleteness of the Weil-Petersson metric for Teichmüller space, Pacific J. Math. 61 (1975), 573-577.

[W4] The length spectra as moduli for compact Riemann surfaces, Ann. of Math. 109 (1979), 323-351.

[W5] On the Weil-Petersson geometry of the moduli space of curves, Amer. J. Math. 107 (1985), 969-997.

Department of Mathematics, Brown University, Providence, RI 02912

E-mail address: daskal@gauss.math.brown.edu

Department of Mathematics, University of California, Irvine, CA 92697

E-mail address: 1katzark@math.uci.edu

Department of Mathematics, University of California, Irvine, CA 92697

E-mail address: raw@math.uci.edu 